\title{
Update to a protocol for a feasibility cluster randomised controlled trial of a peer-led school-based intervention to increase the physical activity of adolescent girls (PLAN-A)
}

Simon J. Sebire ${ }^{1 *}$, Mark J. Edwards', Rona Campbell ${ }^{2}$, Russell Jago ${ }^{1}$, Ruth Kipping ${ }^{2}$, Kathryn Banfield ${ }^{1}$, Bryar Kadir ${ }^{2}$, Kirsty Garfield ${ }^{2}$, Ronan A. Lyons ${ }^{3}$, Peter S. Blair ${ }^{2}$ and William Hollingworth ${ }^{2}$

\begin{abstract}
Background: Physical activity levels are low amongst adolescent girls, and this population faces specific barriers to being active. Peer influences on health behaviours are important in adolescence, and peer-led interventions might hold promise to change behaviour. This paper describes the protocol for a feasibility cluster randomised controlled trial of Peer-Led physical Activity iNtervention for Adolescent girls (PLAN-A), a peer-led intervention aimed at increasing adolescent girls' physical activity levels. In addition, this paper describes an update that has been made to the protocol for the PLAN-A feasibility cluster randomised controlled trial.

Methods/design: A two-arm cluster randomised feasibility trial will be conducted in six secondary schools (intervention $n=4$; control $n=2)$ with year 8 (12-13 years old) girls. The intervention will operate at a year group level and consist of year 8 girls nominating influential peers within their year group to become peer supporters. Approximately $15 \%$ of the cohort will receive 3 days of training about physical activity and interpersonal communication skills. Peer supporters will then informally diffuse messages about physical activity amongst their friends for 10 weeks. Data will be collected at baseline (time 0 (T0)), immediately after the intervention (time 1 (T1)) and 12 months after baseline measures (time 2 (T2)). In this feasibility trial, the primary interest is in the recruitment of schools and participants (both year 8 girls and peer supporters), delivery and receipt of the intervention, data provision rates and identifying the cost categories for future economic analysis. Physical activity will be assessed using 7-day accelerometry, with the likely primary outcome in a fully powered trial being daily minutes of moderate-to-vigorous physical activity. Participants will also complete psychosocial questionnaires at each time point: assessing motivation, self-esteem and peer physical activity norms. Data analysis will be largely descriptive and focus on recruitment, attendance and data provision rates. The findings will inform the sample size required for a definitive trial. A detailed process evaluation using qualitative and quantitative methods will be conducted with a variety of stakeholders (i.e. pupils, parents, teachers and peer-supporter trainers) to identify areas of success and necessary improvements prior to proceeding to a definitive trial.

(Continued on next page)
\end{abstract}

\footnotetext{
*Correspondence: Simon.Sebire@bristol.ac.uk

${ }^{1}$ Centre for Exercise, Nutrition \& Health Sciences, School for Policy Studies,

University of Bristol, Bristol, UK

Full list of author information is available at the end of the article
} 
(Continued from previous page)

Discussion: The study will provide the information necessary to design a fully powered trial should PLAN-A demonstrate evidence of promise. This paper describes an update to the protocol for the PLAN-A feasibility cluster randomised controlled trial related to the data-linkage component.

Trial registration: ISRCTN12543546

Keywords: Physical activity, Peers, Adolescent girls, Intervention, School

\section{Introduction}

The Peer-Led physical Activity iNtervention for Adolescent girls (PLAN-A) study is a feasibility cluster randomised controlled trial of a peer-led intervention which aims to increase adolescent girls' physical activity. The PLAN-A study involves training girls in year 8 of secondary school, who have been nominated by their peers to be "peersupporters", to encourage, facilitate and support their friends' physical activity. The objectives of the feasibility study are to estimate recruitment and data provision rates, examine intervention acceptability, estimate the potential effect of the intervention on increasing girls' moderate-tovigorous physical activity levels, estimate the sample size needed for a definitive trial and examine the consent rate of participants and data custodians for linkage of their study data to academic and health records. The original protocol was published in Pilot and feasibility Studies (https://pilotfeasibilitystudies.biomedcentral.com/ articles/10.1186/s40814-015-0045-8) [1].

\section{Update to data linkage objectives}

Following publication of the original protocol, the study team held discussions with the Institutional Ethics Committee, Trial Steering Committee, Trial Management Group and data linkage experts regarding the data linkage protocol. As a result of these discussions, study objective 8 has been changed and we will now qualitatively examine parents' views regarding allowing their child's data to be used for data linkage with academic records kept by the National Pupil Database (NPD). We will continue to examine the proportion of demographic data (full name, DOB and home postcode) collected from pupils that are of sufficient quality and completeness to link to educational attainment data held by the NPD. As asking participants and parents to consent to a hypothetical data linkage scenario was deemed to be too complex and not likely to accurately reflect responses to a real request for consent, the original proposal to seek hypothetical consent from parents, data custodians and local authorities to take part in data linkage has been changed. We will now include questions about data linkage in interviews with parents of participants. In the interviews, we will qualitatively explore parents' understanding of data linkage, their concerns and the length of time that they would like their consent to link study data to remain in place. Parents will be asked to comment on their views on allowing their child's study data to be linked to educational data kept by the NPD. In addition, senior school management in each school will be asked whether they would consent to study participants' data being used for educational data linkage. The NPD administrators will be asked if they would hypothetically allow a future trial of PLAN-A to link pupil data with attainment and absence data they hold. All changes have been agreed by the Research Ethics Committee of the School for Policy Studies at the University of Bristol, the independent Trial Steering Committee and the project funder.

\section{Acknowledgements}

The work was undertaken with the support of the Centre for the Development and Evaluation of Complex Interventions for Public Health Improvement (DECIPHer), a UKCRC Public Health Research Centre of Excellence. Joint funding (MR/KO232331/1) from the British Heart Foundation, Cancer Research UK, Economic and Social Research Council, Medical Research Council, the Welsh Government and the Wellcome Trust, under the auspices of the UK Clinical Research Collaboration, is gratefully acknowledged. This study was designed and delivered in collaboration with the Bristol Randomised Trials Collaboration (BRTC), a UKCRC Registered Clinical Trials Unit in receipt of National Institute for Health Research CTU support funding. None of the funders had involvement in the Trial Steering Committee, the data analysis, data interpretation, data collection or writing of the paper. The views expressed in this publication are those of the authors and not necessarily any of the funding bodies listed here.

RAL receives funding through the Farr Institute of Health Informatics Research. The Farr Institute is supported by a consortium of ten UK research organisations: Arthritis Research UK, the British Heart Foundation, Cancer Research UK, the Economic and Social Research Council, the Engineering and Physical Sciences Research Council, the Medical Research Council, the National Institute of Health Research, the National Institute for Social Care and Health Research (Welsh Government), the Chief Scientist Office (Scottish Government Health Directorates) and Wellcome Trust. MRC Grant No MR/K006525/1. The sponsor of this study is the University of Bristol, Research and Enterprise Development, 3rd Floor, Senate House, Tyndall Avenue, Bristol, BS8 1TH, UK www.bristol.ac.uk/red/.

\section{Funding}

This project was funded by the National Institute for Health Research (Public Health Research Programme) (project number 13/90/16). The views and opinions expressed therein are those of the authors and do not necessarily reflect those of the Public Health Research Programme, NIHR, NHS or the Department of Health.

\section{Availability of data and materials} Not applicable.

\section{Authors' contributions}

The study was conceived by SJS, RJ, RC, WH, RK, PB and RAL. MJE is the Project Manager, KB is the qualitative Research Assistant, KG is the health economics analyst and BK is the project statistician. The first draft of this manuscript was written by SJS with input from all other authors. All authors have edited and critically reviewed the paper for intellectual content and approved the final version of the paper. 


\section{Competing interests}

$\mathrm{RC}$ is a board member of DECIPHer Impact, a not for profit company that sells the DECIPHer-ASSIST smoking prevention programme (formerly known as ASSIST). There are no further competing interests to declare.

\section{Consent for publication}

Not applicable.

\section{Ethics approval and consent to participate}

This update has received approval from the University of Bristol's School for Policy Studies Ethics Committee (SPSREC14 15.A50). All parents taking part in an interview will provide informed consent.

\section{Author details}

${ }^{1}$ Centre for Exercise, Nutrition \& Health Sciences, School for Policy Studies, University of Bristol, Bristol, UK. ${ }^{2}$ School of Social \& Community Medicine, University of Bristol, Bristol, UK. ${ }^{3}$ Farr Institute, Swansea University Medical

School, Swansea, UK.

Received: 14 September 2016 Accepted: 16 November 2016

Published online: 01 December 2016

\section{Reference}

1. Sebire SJ, et al. Protocol for a feasibility cluster randomised controlled trial of a peer-led school-based intervention to increase the physical activity of adolescent girls (PLAN-A). Pilot and Feasibility Studies. 2016;2:2.

Submit your next manuscript to BioMed Central and we will help you at every step:

- We accept pre-submission inquiries

- Our selector tool helps you to find the most relevant journal

- We provide round the clock customer support

- Convenient online submission

- Thorough peer review

- Inclusion in PubMed and all major indexing services

- Maximum visibility for your research

Submit your manuscript at www.biomedcentral.com/submit 